Supporting Information

\title{
Stretchable and Anisotropic Conductive Composite Hydrogel as Therapeutic Cardiac Patches
}

Chenlong Wang, $¥$ Yi Chai, $₫$ Xiaodong Wen, $₫$ Yongjian Ai, He Zhao, Wanting Hu, Xiaoping Yang, Ming-Yu Ding, Xiaolu Shi, Qingfei Liu, Qionglin Liang*

\section{Supplemental Experimental Procedures}

All chemicals used were of analytical grade without any further purification. All aqueous solutions were freshly prepared with high purity water.

Synthesis of Polypyrrole nanotubes: PPy nanotubes with uniform diameters were first synthesized using oxidation polymerization with pyrrole, methyl orange (MO) and $\mathrm{FeCl}_{3}$ as material, dopant and oxidant, respectively, a previously reported method ${ }^{1}$ with slight modifications. In this procedure, $\mathrm{FeCl}_{3}$ and $\mathrm{MO}$ can form supramolecular clusters in solutions, acting as a reactive self-degraded template, directing the growth of PPy on its surface and promoting them assemble into tubular structures ${ }^{2}$. In-situ sulfonates doping polymerization can oxidize high-energy electron and create radical cation ${ }^{3-5}$. Polypyrrole nanotubes was synthesized by self-degraded template method, modified from a previous study ${ }^{6}$. Put $250 \mathrm{mM}$ of ferric chloride $\left(\mathrm{FeCl}_{3}\right.$, Sigma Aldrich) and 5 $\mathrm{mM}$ methyl orange (MO, Sigma Aldrich) in $300 \mathrm{~mL}$ of deionized water, and stirred for $5 \mathrm{~min}$. Then $50 \mathrm{mM}$ pyrrole monomer was added dropwise and the mixture was stirred at room temperature for $24 \mathrm{~h}$. When the reaction finished, the produced PPy nanotubes 
precipitate was washed with ethanol/deionized water for several times until filtrate was neutral and colorless. The product was finally dried under a vacuum atmosphere and the temperature was keep at $60^{\circ} \mathrm{C}$ for $24 \mathrm{~h}$.

Preparation of PPy nanotubes: A $0.1 \mathrm{~g}$ sample of PPy nanotubes was added into 10 $\mathrm{mL}$ sodium alginate (SA, Sigma Aldrich) aqueous solution ( $0.4 \mathrm{~g}$ of $\mathrm{SA})$. Then, the mixture was ultrasonic dispersed under Ultrasonic Cell Disruption System (XINZHI, SCIENTZ- II D, China) for $1 \mathrm{~h}$. The procedure was as follows: sixty percent of the maximum power, worked every $3 \mathrm{~s}$ accompany with rest $2 \mathrm{~s}$. The dispersion of the PPy nanotubes was stored at $4{ }^{\circ} \mathrm{C}$.

Fabrication of PPy nanotube Hydrogels: Firstly, $1.244 \mathrm{~g}$ of acrylamide (AM; Sigma Aldrich) was added into $5 \mathrm{~mL}$ of deionized water along with $5 \mathrm{mg} \mathrm{N}$, N'- methylene bisacrylamide (MBAA, Sigma Aldrich), and the mixture was stirred for $10 \mathrm{~min}$. Next, PPy nanotubes dispersion of $0,0.1,0.5,1$ and $2 \mathrm{~mL}$ were add to the mixture. SA solution of $4 \mathrm{w} / \mathrm{v} \%$ with $5,4.9,4.5,4$ and $3 \mathrm{~mL}$ were added afterward and the mixture was degassed in a vacuum chamber for $30 \mathrm{~min}$. Then, Ammonium persulfate (APS, Sigma Aldrich) of $0.02 \mathrm{~g}$ and $2 \mu \mathrm{L}$ Tetramethylethylenediamine (TEMED, Sigma Aldrich) were injected and mixed by ultrasound, and the mixture was poured into poured into a glass mold $(75.0 \mathrm{~mm} \times 50.0 \mathrm{~mm} \times 0.75 \mathrm{~mm})$ at $50{ }^{\circ} \mathrm{C}$ for $20 \mathrm{~min}$ to keep the synthesis reaction. Then, the hydrogel was soaked in the $50 \mathrm{mM} \mathrm{CaCl}_{2}$ (Sigma Aldrich) solution for $24 \mathrm{~h}$ to generate PNHs. The PNHs were soaked by fresh high-purity water for $24 \mathrm{~h}$ to wipe out the unreacted monomer. Before the measurement, hydrogels were fully hydrated in $50 \mathrm{mM} \mathrm{CaCl}_{2}$ to guarantee the uniqueness of the variable. 
Mechanical characteristic analyses and fabrication of A-PNHs: Tensile testing of the hydrogel was performed using a previously described method $^{7}$. In brief, $20.0 \mathrm{~mm} \times 10.0$ $\mathrm{mm} \times 0.75 \mathrm{~mm}$ rectangular strips of the patches from the 3 groups were strained to the breaking point at a constant crosshead speed of $5 \mathrm{~mm} / \mathrm{min}$ using an INSTRON 5842 mechanical testing machine (INSTRON Inc., Canton, USA) and analyzed for the breaking stress and strain using the software package Merlin (INSTRON Inc.). Then, PNHs were circularly strained before the breaking point at a constant crosshead speed of $5 \mathrm{~mm} / \mathrm{min}$. The stretch ratio $\lambda=5$. After straining enough cycles, A-PNHs hydrogel can be obtained. The produced A-PNHs hydrogels were stored at $4{ }^{\circ} \mathrm{C}$ to prevent dehydration. The rheological measurement was also performed by Aaton Paar Physica MCR301. The diameter of the steel plate was $25 \mathrm{~mm}$ with a gap of $1 \mathrm{~mm}$. The G' (storage modulus) and G" (loss modulus) of the hydrogels were calculated by taking the values at a particular angular frequency of $10 \mathrm{rad} / \mathrm{s}$ for the oscillation mode at room temperature and the shear stress increased from 1 to $10000 \mathrm{~Pa}$.

Patch conductivity measurement: Before the measurement, hydrogels were also fully hydrated in $50 \mathrm{mM} \mathrm{CaCl}_{2}$ to guarantee the uniqueness of the variable. The conductivity of the cardiac patch was evaluated using a four-probe conductive analyzer (HewlettPackard Development Company, Palo Alto, USA). The double network hydrogel, PNH or A-PNH patches were placed on a platform and the probes were placed at a $7 \mathrm{~mm}$ distance for conductivity measurements using a linear double-sweep model. The voltage increased from -5 to $+5 \mathrm{~V}$ in $100 \mathrm{mV}$ increments (compline $10 \mathrm{~mA}$ for $2 \mathrm{~s}$, delay time is $100 \mu \mathrm{sec})$. The conductivity was calculated according to the slopes. 
Orientation analyses: Firstly, the PPy nanotubes in nanocomposite after different drawing cycles $(0,20,60,100,140$ and 200 cycles $)$ were imaged. To avoid background signals from blurring PPy nanotubes of hydrogels, images were observed under confocal microscopy (ECLIPSE Ni-E; Nikon, Tokyo, Japan) with 20X objective (N.A. 0.75) and recorded with sCMOS camera (Neo sCMOS, DC-152Q-C00-FI; Andor, Belfast, UK) from the surface to the center of nanocomposite $(0-180 \mu \mathrm{m}$, step size is $15 \mu \mathrm{m}$ ). Images were processed using ImageJ (Version 1.52n 22 March 2019, https://imagej.nih.gov/ij/). For direction measurement, the brightness and contrast of the images were adjusted to facilitate the next step. The boundaries of PPy nanotubes were obtained by detecting the threshold of the images, removing small spots from background noise. The center position and direction of PPy nanotubes in every frame were identified by analysis the directionality of boundaries with local gradient direction algorithm. The angle was 0 degree if parallel to the horizontal direction and \pm 90 degrees if perpendicular to the horizontal direction. We counted the direction of more than 500 nanotubes in every drawing cycle. The confocal microscopy images of different layers under different drawing cycles were analyzed by ImagePro Plus software. To describe the changing characteristics, we measured every angle between PPy nanotube and the drawing direction and visualized the statistics.

Experimental animals: Sprague-Dawley (SD) rats [neonatal or adult (weighing 225$250 \mathrm{~g})$ ] were obtained from Laboratory Animal Research Center, Tsinghua University (Beijing, China). All animal protocols and procedures were approved by the Ethics 
Review Committee on Animal Experimentation of Tsinghua University (Beijing, China). The approval accreditation number was 16-LQL1 and 18-LGA1.

Isolation and Culture of Neonatal Rat Cardiomyocytes: Neonatal rat cardiomyocytes were isolated and cultured according to a previous study ${ }^{8}$. All steps and materials were the same with their protocols. In brief, rat hearts were extracted from the body with curved scissors and transferred immediately into the bacterial dish containing $1 \times \mathrm{PBS}$ (without $\mathrm{Ca}^{2+}, \mathrm{Mg}^{2+}$ ) (cellgro) with $20 \mathrm{mM}$ 2,3-Butanedione monoxime (BDM, Sigma Aldrich). Then, transfer cleaned hearts into a drop of $0.0125 \%$ trypsin in HBSS isolation medium (approximately $250 \mu \mathrm{L}$ ) in a third bacterial dish (on ice) and used the curved scissors to mince hearts into small pieces (approximately $0.5-1 \mathrm{~mm}^{3}$ ). Transfer minced hearts into a conical tube containing $10 \mathrm{~mL}$ of the isolation medium (on ice) and incubated with gentle agitation at $4{ }^{\circ} \mathrm{C}$ for an overnight. Later, $15 \mathrm{mg}$ of collagenase/dispase mixture (Roche) was dissolved in $10 \mathrm{~mL}$ L15-medium(cellgro), supplemented with $20 \mathrm{mM}$ BDM (digestion medium). The tissue pieces were digested by the enzyme solution. Cells seeded into $10 \mathrm{~cm}$ cell culture dishes and incubated for 1-3 $\mathrm{h}$ in cell culture incubator. This pre-plating step removed fibroblasts and endothelial cells, which would adhere to the uncoated cell-culture dish. After incubation, washed non-adherent cardiomyocytes from $10 \mathrm{~cm}$ culture dish for cytocompatibility assay. Cytocompatibility assay: A LIVE/DEAD assay (Invitrogen, Life Technologies, Grand Island, NY) was used to quantify the viability of the cells in different soaking media. Briefly, cells were incubated at room temperature for $20 \mathrm{~min}$ in a $10 \mathrm{~mL}$ HBSS solution of calcein-AM $(100 \mu \mathrm{L})$ and ethidium homodimer-1 $(10 \mu \mathrm{L})$. Then, the solution was 
aspirated, and cells were rinsed with PBS and developed using fluorescence microscopy. Live cells were characterized by intracellular esterase activity which cleaves the calcein-AM to form fluorescent green calcein. Dead cells were reported by the presence of red fluorescent ethidium homodimer-1, which entered through the ruptured cell membrane and bound to nucleic acids in the nucleus. Fluorescent images were obtained and analyzed using ImageJ software.

Cell orientation: CMs with a concentration of $4 \times 10^{4}$ cells $/ \mathrm{mL}(1 \mathrm{~mL})$ were seeded on the different substrates $(2 \mathrm{~cm} \times 2 \mathrm{~cm})$ in six-hole plate. This cell-laden hydrogel patch was also used in vivo experiments. Cell orientation was evaluated using confocal microscopy by staining CM marker sarcomeric $\alpha$-actinin at 7 days culture. To perform immunofluorescent staining, CMs seeded were rinsed and fixed in $4 \%$ paraformaldehyde (PF) in Dulbecco's Phosphate Buffered Saline (DPBS) for 15 min at room temperature and permeabilized with $0.3 \%$ Triton $\mathrm{X}-100$ for $10 \mathrm{~min}$ at room temperature. Then, the samples were blocked in 10\% goat serum (GS) and were soaked ( $4{ }^{\circ} \mathrm{C}$, overnight) in 1:200 dilutions of primary antibodies specific to sarcomeric aactinin (ab9465, abcam, USA) in 10\% GS. The samples were subsequently treated with secondary antibodies (1:1000 dilution) (ab150113, abcam, USA) for $60 \mathrm{~min}$ at room temperature. Then, the samples were soaked in 4',6-diamidino-2-phenylindole (DAPI) in 1:1000 dilutions for cell nuclei for $15 \mathrm{~min}$. Fluorescent images were obtained and analyzed using ImageJ software.

Demonstration of the nanocomposite as patch in vivo: Rat models of acute myocardial infarction were established by a previously reported method $^{9}$ with slight modifications. 
At the beginning, adult SD female rats were anesthetized, intubated and ventilated with 2 to $2.5 \%$ isoflurane mixed with oxygen. Rat models' fourth intercostal space was exposed, and the left anterior descending coronary artery was ligatured for $60 \mathrm{~min}$. ECG was monitored before and after myocardial damage at different time points, and the millivolt of ST and ST-T segment were measured simultaneously. Then, the damaged rat hearts were patched with pure double network hydrogels, PNHs or A-PNHs and ECG were measured.

Inflammatory experiment: The examination of inflammatory response was performed using ELISA kits (Meimian Biotechnology, Yancheng, Jiangsu, China) to quantify the serum inflammatory factor levels of CD3, CD8, and CD68. Rats were distributed into 2 following groups: Patched and MI only as control group $(n=3)$. Serum samples were collected for the experiment according to the manufacturer's instructions at 7 days postoperation. Subsequently, the absorbance values of ELISA plates were measured by a microplate reader (Infinite F50, Tecan, Männedorf, Switzerland).

Histological analysis: The myocardial tissues were fixed in 10\% neutral formaldehyde solution for $24 \mathrm{~h}$, then they were embedded, sliced, and stained with hematoxylin-eosin (HE) and Masson Trichrome. All samples were examined using an optical microscope (Olympus Optical Co., Tokyo, Japan).

Statistical analyses: Data are presented as the means \pm standard error of means unless otherwise indicated. Analyses are performed using SPSS software (19.0), with the critical $\alpha$-level set at $p<0.05$. Comparisons among multiple groups are made using one-way analysis of variance (ANOVA). 


\section{Figures S1-S14 and Table S1}

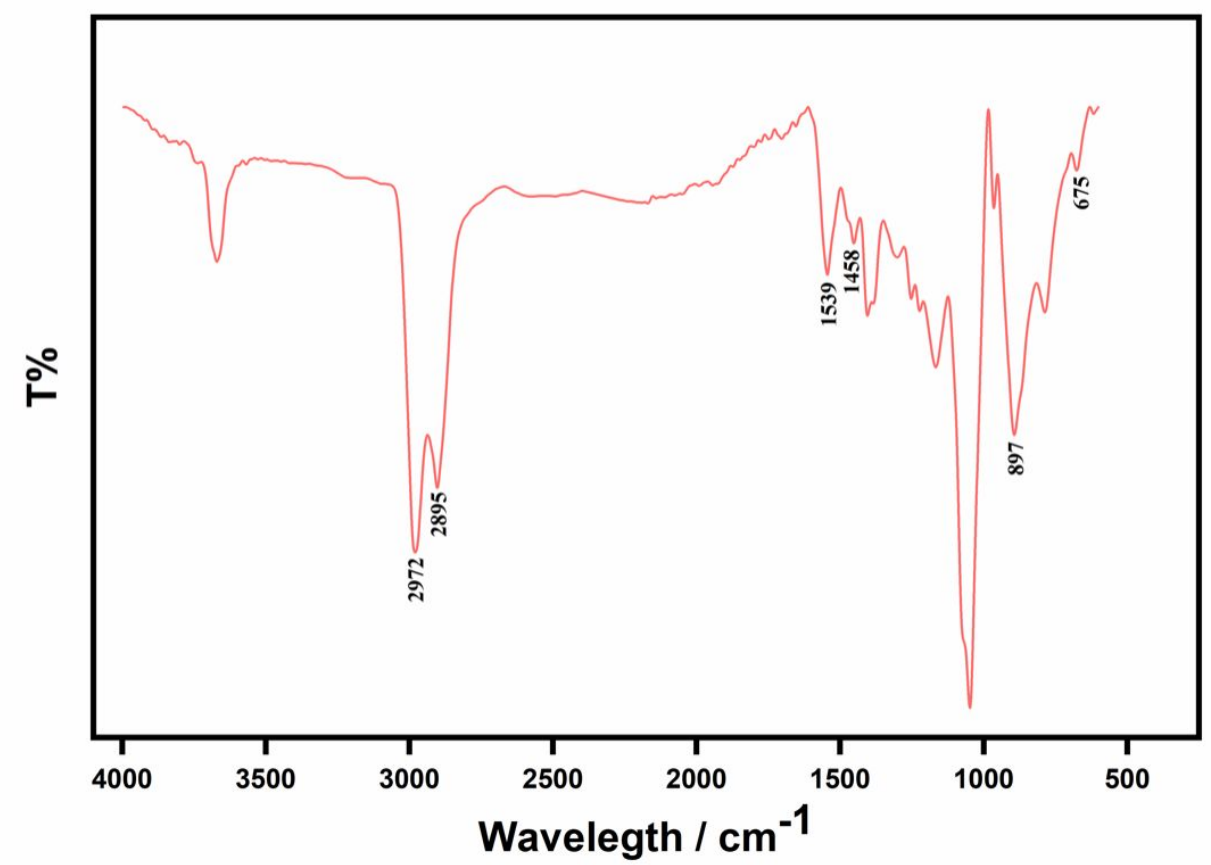

Figure S1. The FTIR spectra of PPy nanotubes.
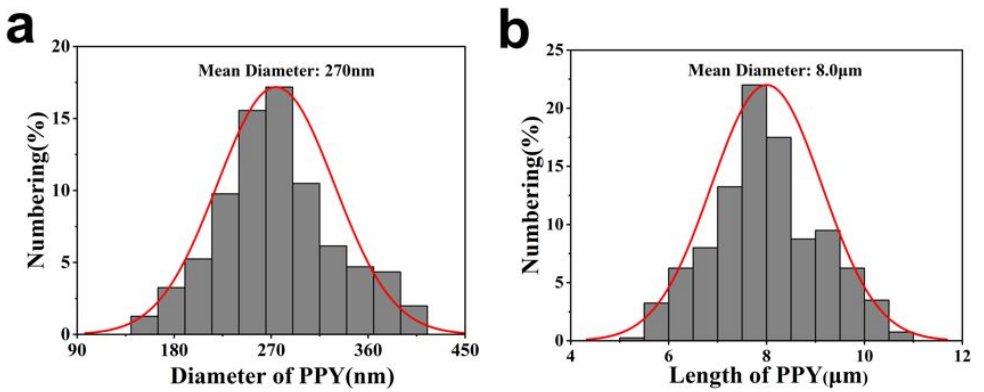

Figure S2. The statistics of PPy nanotubes size distribution. a) The diameter and b) the length of the PPy nanotubes. 


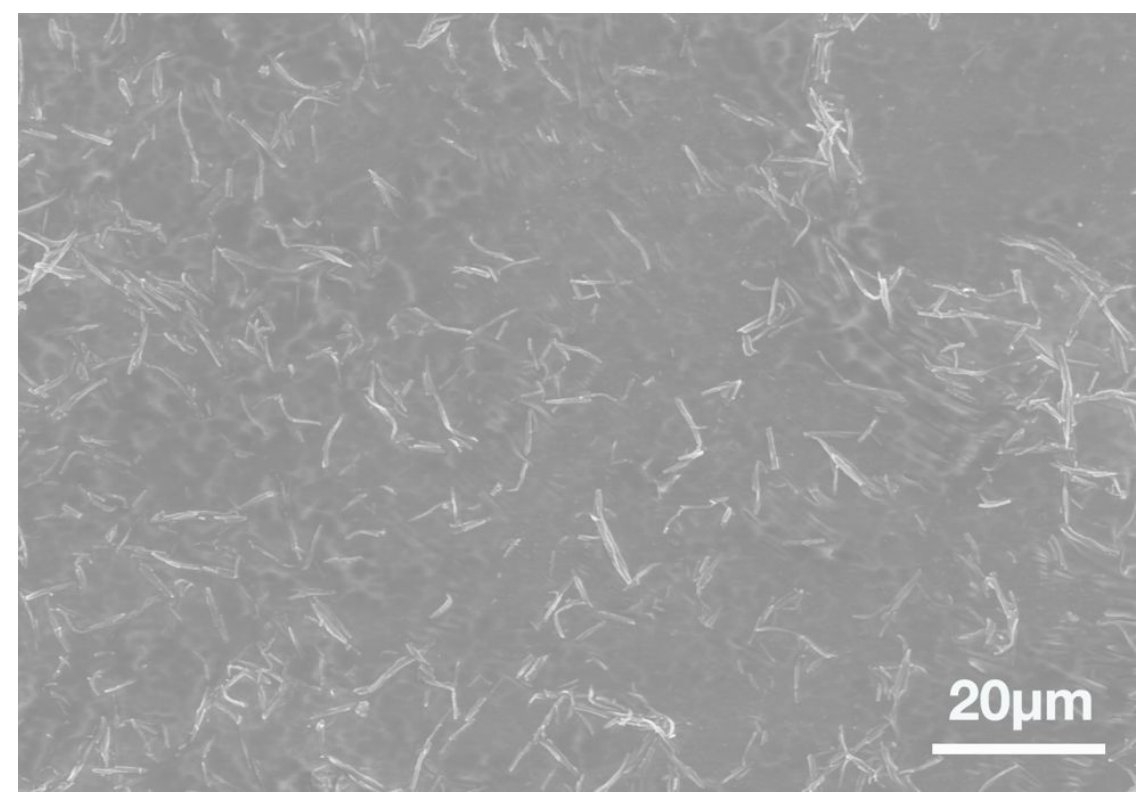

Figure S3. The SEM image of PPy nanotubes dispersed in the sodium alginate solution.

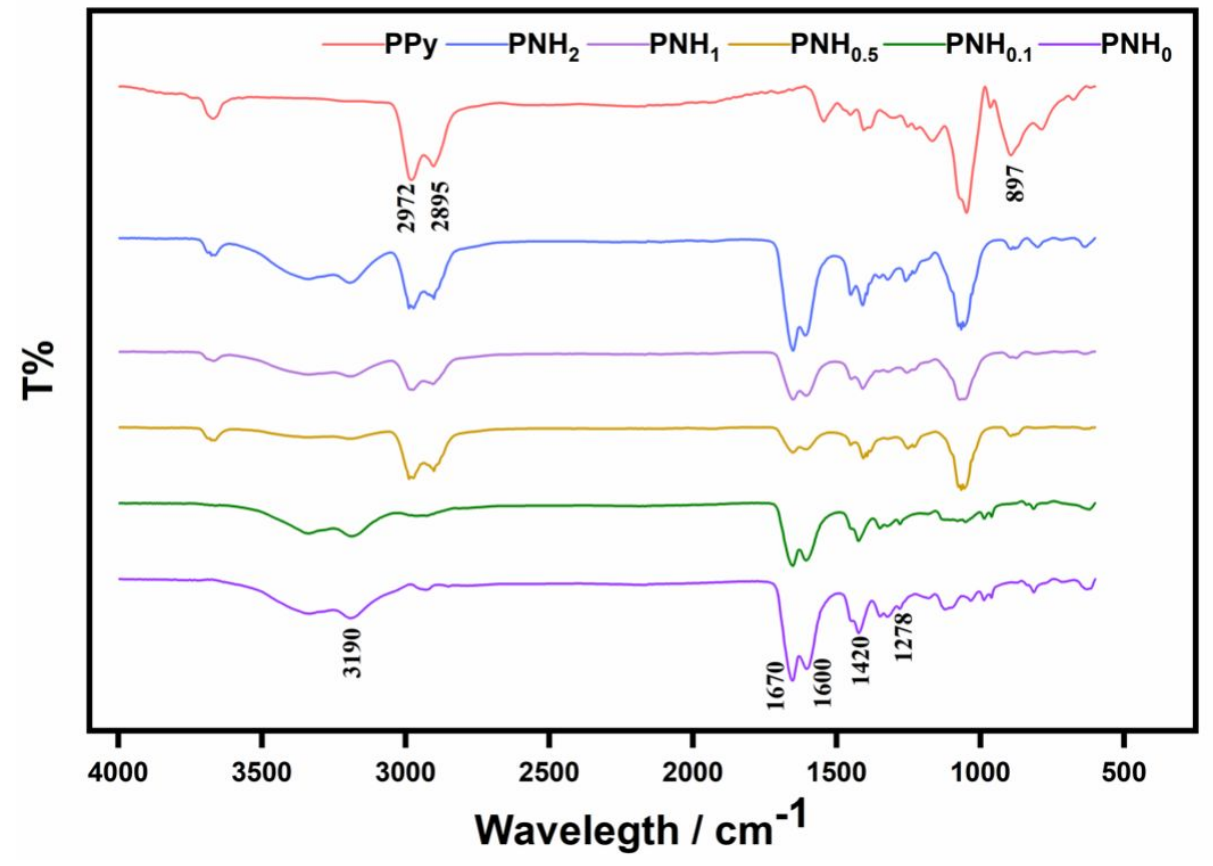

Figure S4. The FTIR spectra of PPy nanotubes and $\mathrm{PNH}_{\mathrm{x}}$. 


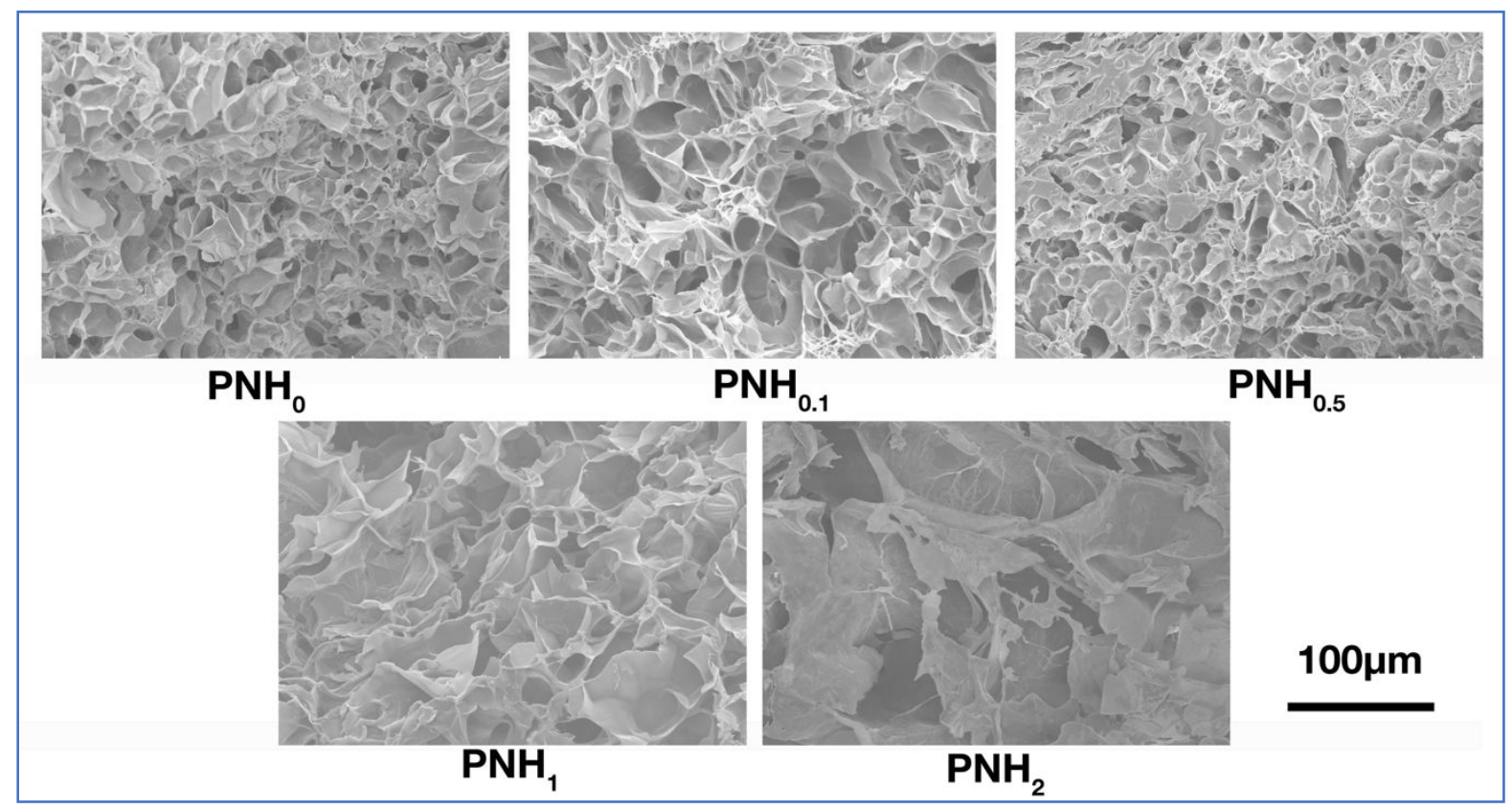

Figure S5. The SEM image of $\mathrm{PNH}_{\mathrm{x}}$ 's structure.

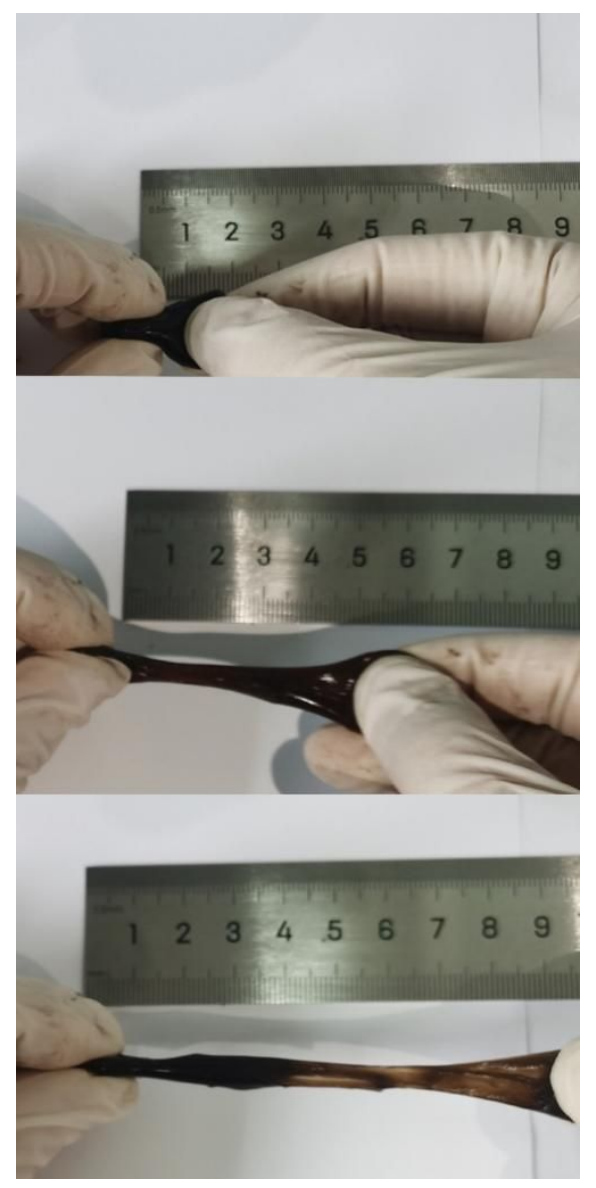

Figure S6. The tensile property of $\mathrm{PNH}_{1}$. 


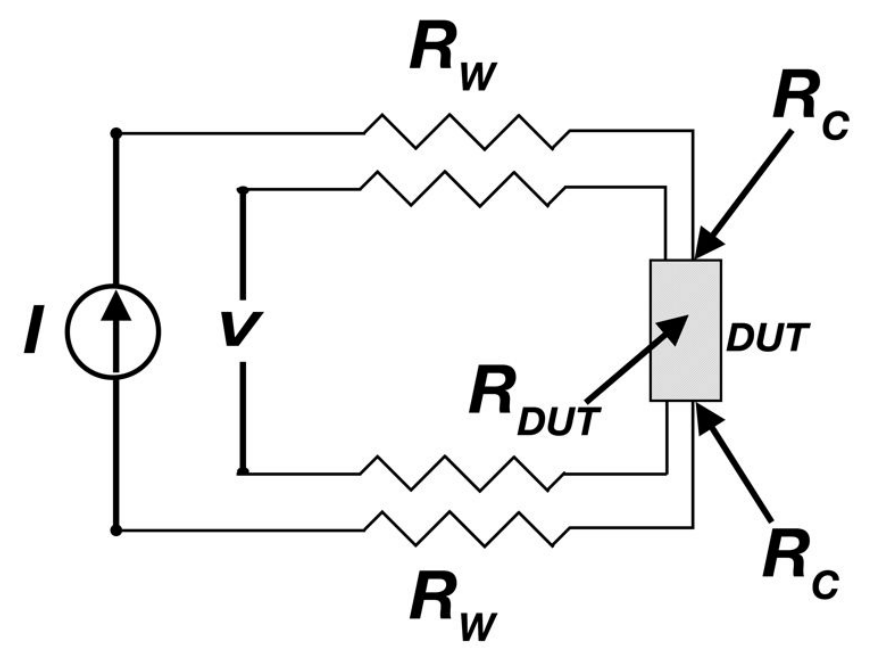

Figure S7. The schematic diagram of four-probe electrical resistivity measuring technique.
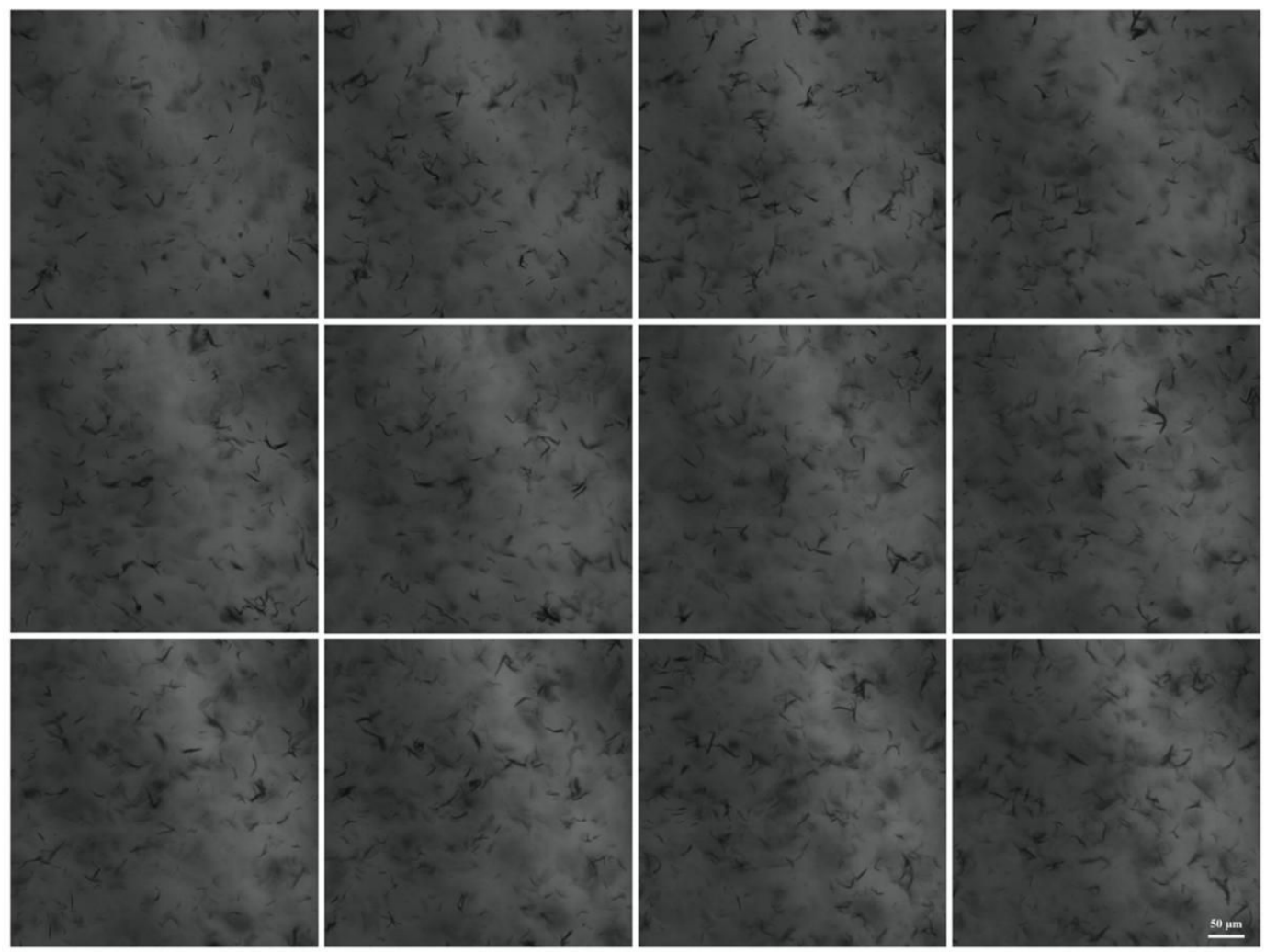

Figure S8. Confocal microscopy images from the surface to the center of PNH (0 $\mu \mathrm{m}-180 \mu \mathrm{m}$, step size is $15 \mu \mathrm{m}$ ). 

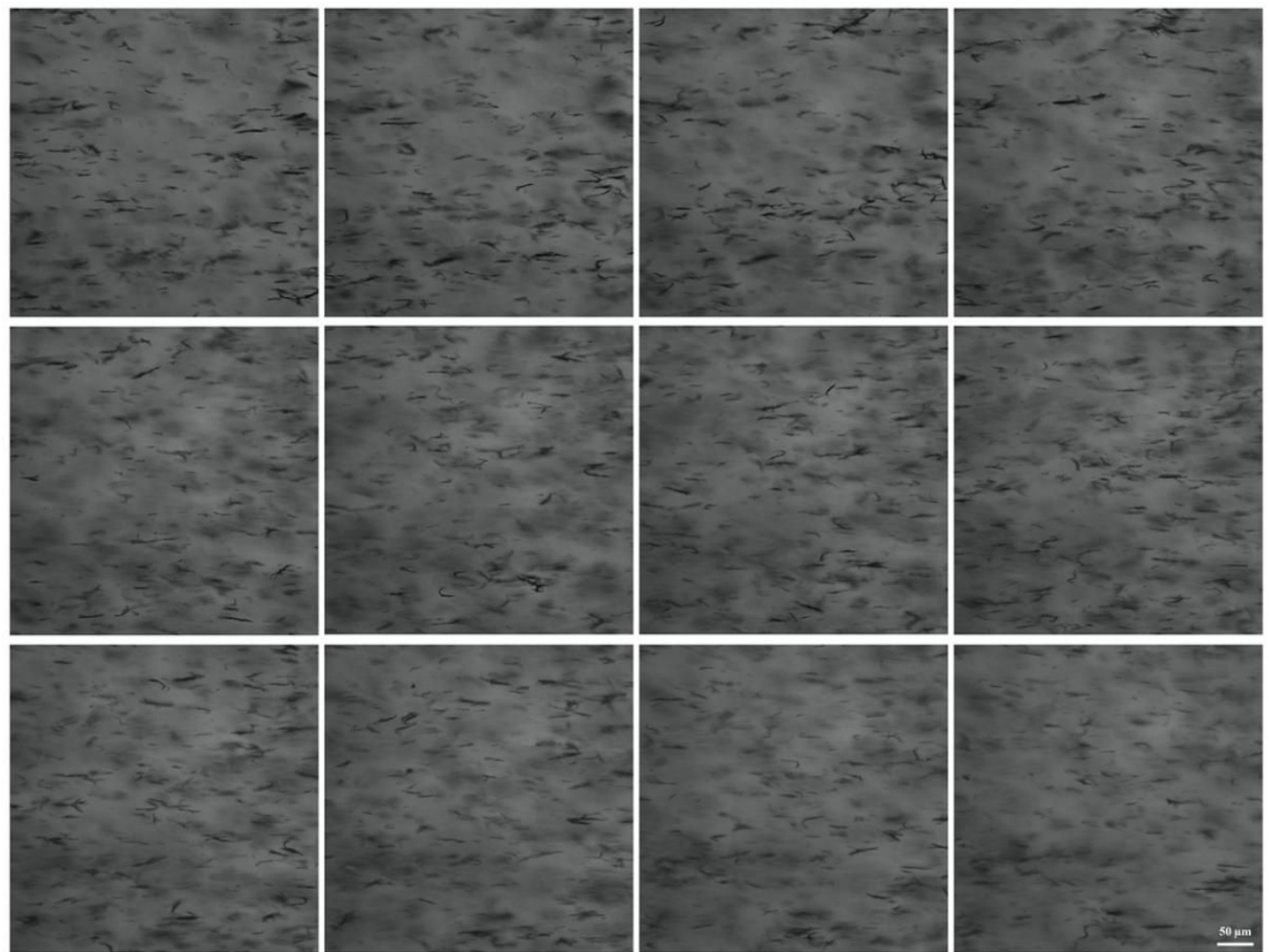

Figure S9. Confocal microscopy images from the surface to the center of A-PNH (0 $\mu \mathrm{m}-180 \mu \mathrm{m}$, step size is $15 \mu \mathrm{m})$.

a

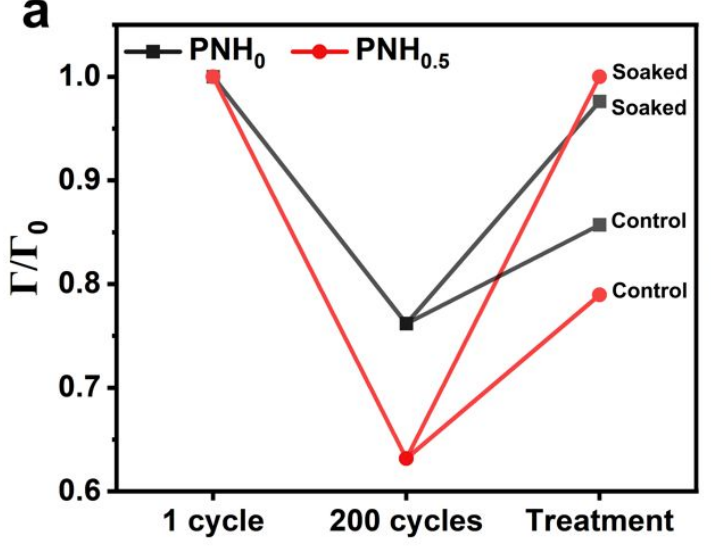

b

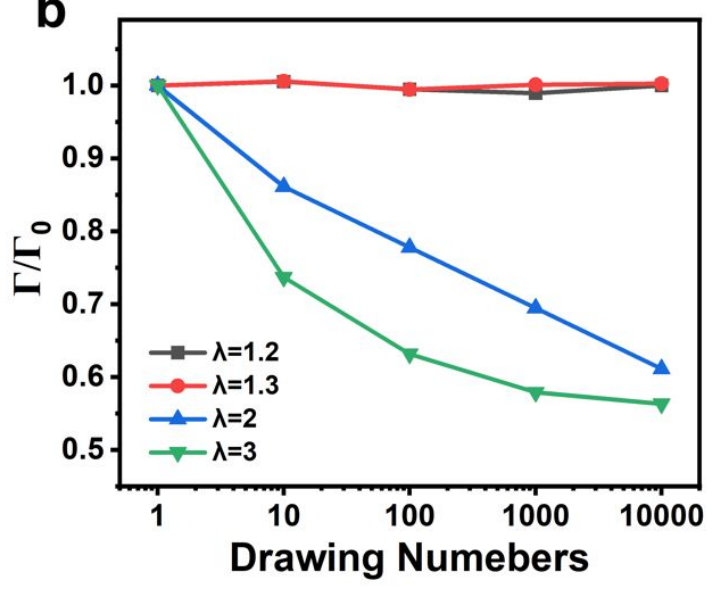

Figure S10. a) The Young's modulus $\Gamma$ under different conditions contrast with the Young's modulus on the first loading $\Gamma_{0}$. These ionic bonds can reconstruct with time and $\mathrm{Ca}^{2+}$ socked. Hydrogels which left for 1 day were designed as control. The stretch ratio $\lambda=5$. b) The Young's modulus $\Gamma$ of PNHs with different stretch ratio $\lambda$. 


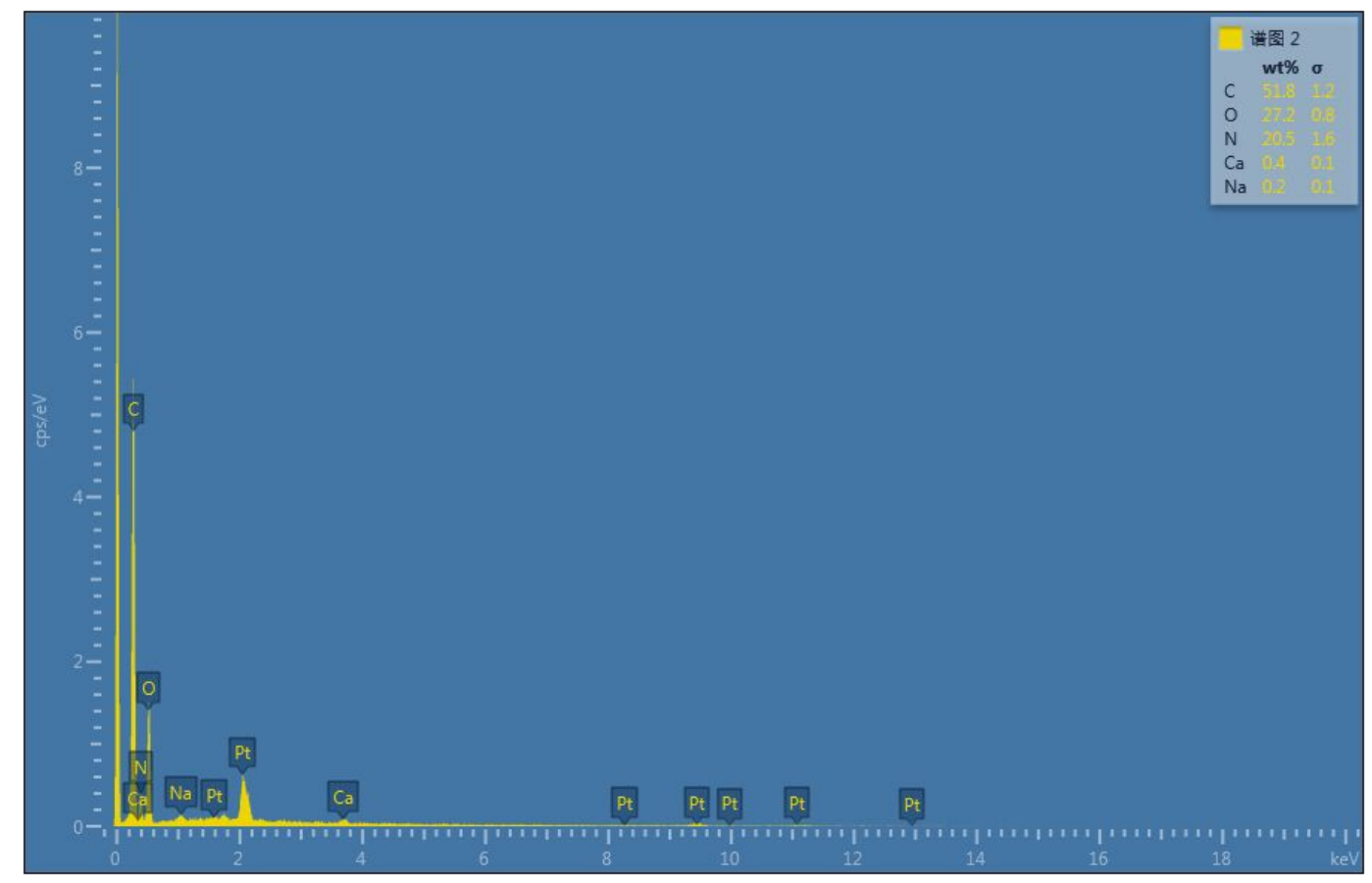

Figure S11. The energy dispersive X-ray spectroscopy (EDX) image of the pure $\mathrm{PNH}_{0}$.

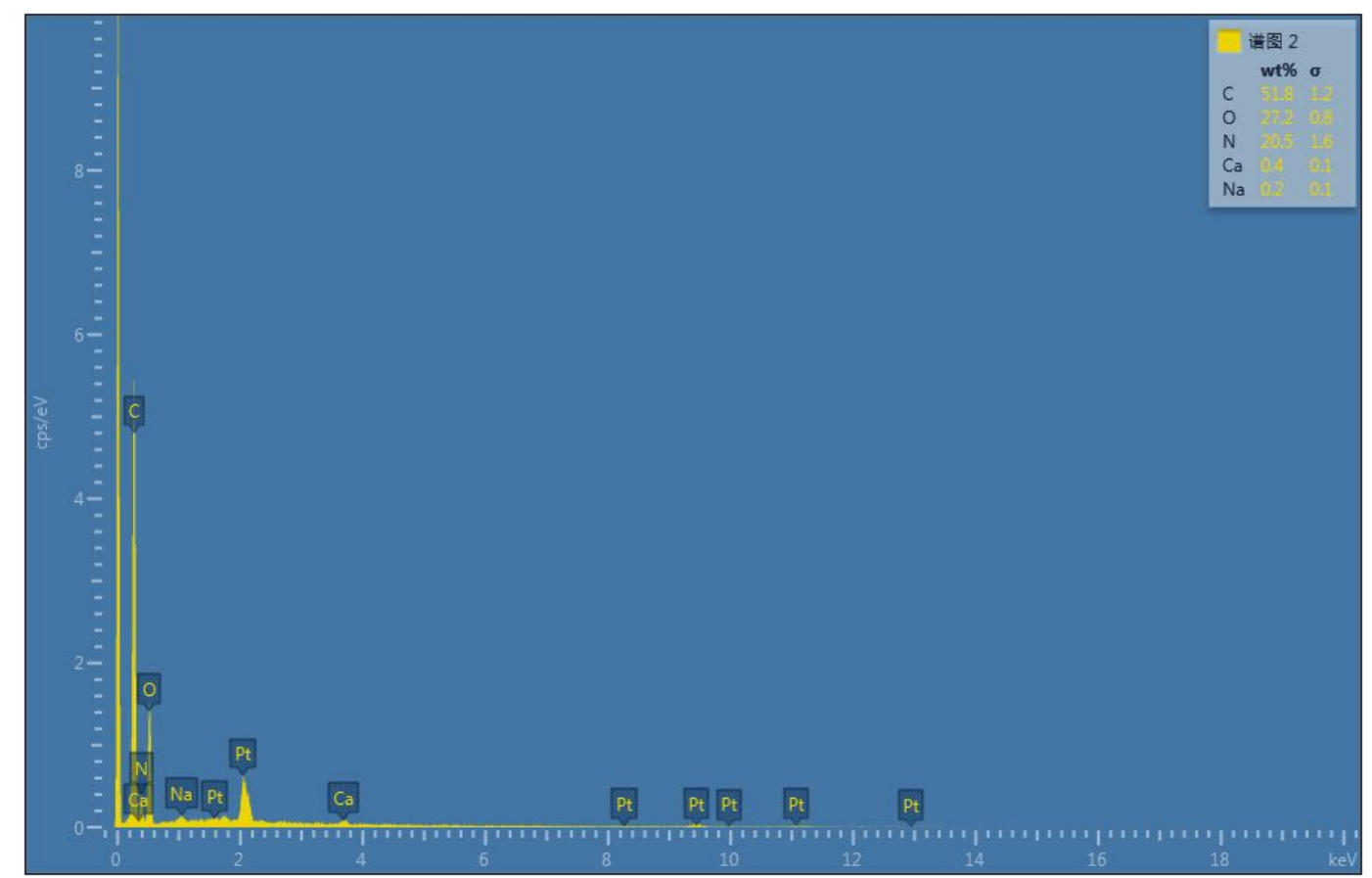

Figure S12. The EDX image of the pure A-PNH. 

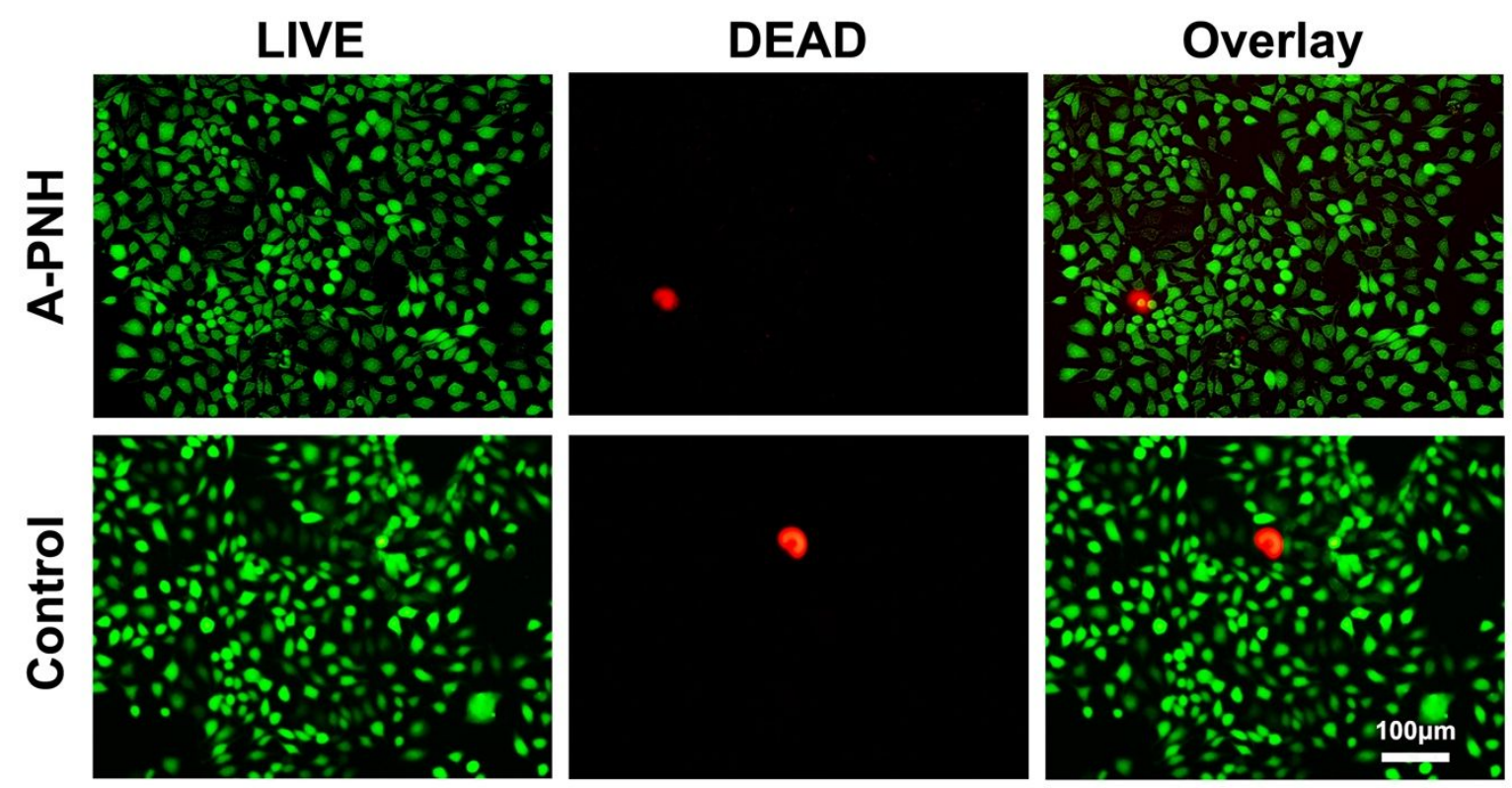

Figure S13. Living/dead staining of the cardiomyocytes of cardiac muscle cells (CMs) cultured for $72 \mathrm{~h}$ in 7 days gel-conditioned media. Off-soaked media as control. Green: live cells, red: dead cells.
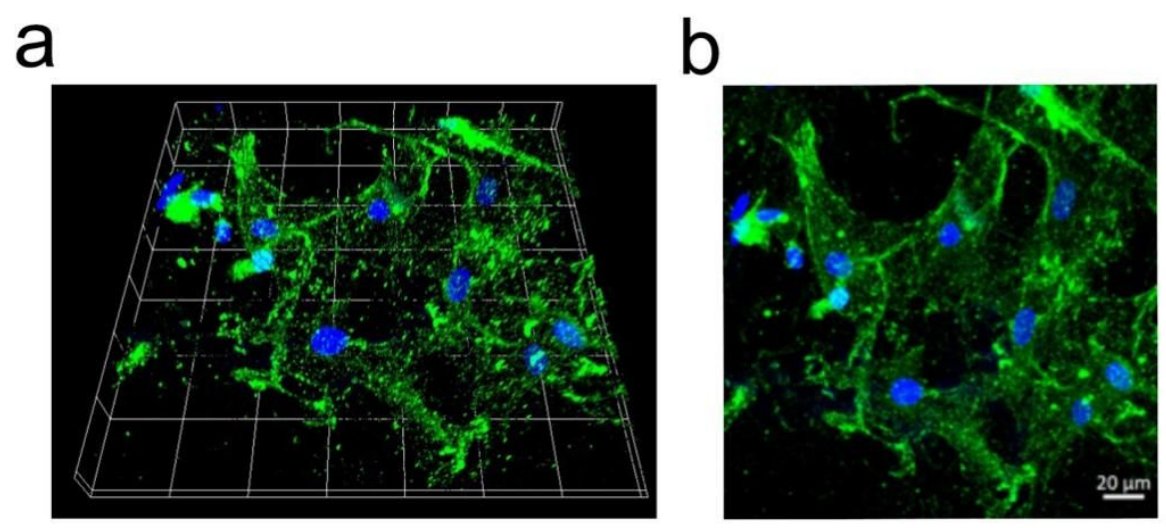

Figure S14. a) Three-dimensional reconstruction and b) representative confocal images indicating that CMs grown on the $\mathrm{PNH}$ extended randomly after 7 days of culture. 


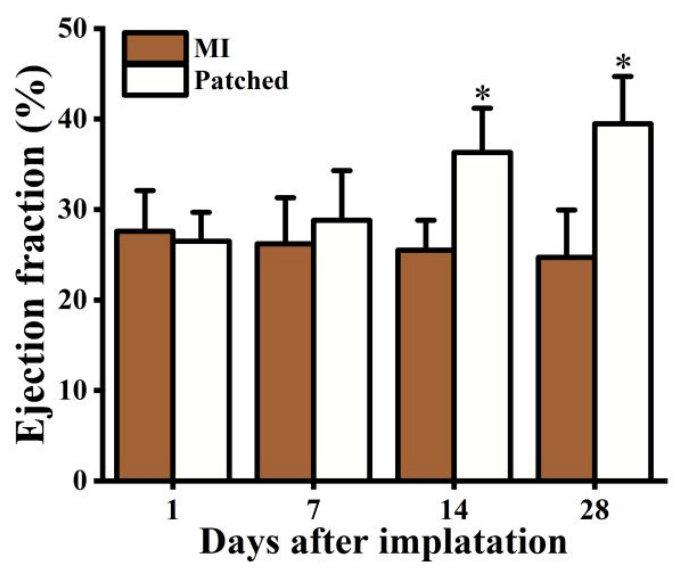

Figure S15. Left ventricle cardiac function with different days measured by longitudinal echocardiography. Rats are randomly divided into 2 groups:MI only and explanted A-PNH patches. Compared to MI only hearts after 14 and 28 days implantation, A-PNH patch treatment led to a significantly improved. Data are analyzed using one-way ANOVA. Significance is set at $p<0.05$ versus control. ( $n=$ 6).

Table S1. Fe element content analyzed by Inductively Coupled Plasma Atomic Emission Spectroscopy.

\begin{tabular}{cc}
\hline $\mathrm{Fe}$ & $\mathrm{wt} \%$ \\
\hline $\mathrm{PNH}_{0}$ & 0.0013 \\
$\mathrm{~A}-\mathrm{PNH}$ & 0.0029 \\
\hline
\end{tabular}

\section{Supplemental References}

1. Yan, Y., Facile Synthesis of Polypyrrole Nanotubes and Their Supercapacitive Application. Int. J. Electrochem. Sci. 2017, 12, 9320-9334.

2. Yang, X.; Zhu, Z.; Dai, T.; Lu, Y., Facile Fabrication of Functional Polypyrrole Nanotubes via a Reactive Self-Degraded Template. Macromol. Rapid Commun. 2005, 26, 1736-1740.

3. Chen, W.; Wan, X.; Xu, N.; Xue, G., Ordered Conducting Polypyrrole Doped with Sulfopropyl Ether of $\beta$-Cyclodextrin. Macromolecules 2003, 36, 276-278. 
4. Shi, H. Y.; Ye, Y. J.; Liu, K.; Song, Y.; Sun, X., A Long-Cycle-Life Self-Doped Polyaniline Cathode for Rechargeable Aqueous Zinc Batteries. Angew Chem Int Ed Engl 2018, 57, 16359-16363.

5. Lombardo, V.; D'Urso, L.; Mannino, G.; Scalese, S.; Spucches, D.; La Magna, A.; Terrasi, A.; Puglisi, R. A., Transparent conductive polymer obtained by in-solution doping of PEDOT:PSS. Polymer 2018, 155, 199-207.

6. Sapurina, I.; Li, Y.; Alekseeva, E.; Bober, P.; Trchová, M.; Morávková, Z.; Stejskal, J., Polypyrrole nanotubes: The tuning of morphology and conductivity. Polymer 2017, $113,247-258$.

7. Zhang, F.; Xiong, L.; Ai, Y.; Liang, Z.; Liang, Q., Stretchable Multiresponsive Hydrogel with Actuatable, Shape Memory, and Self-Healing Properties. Adv. Sci. 2018, $5,1800450-1800456$.

8. Ehler, E.; Moore-Morris, T.; Lange, S., Isolation and culture of neonatal mouse cardiomyocytes. J. Vis. Exp. 2013, 79, e50154-e50164.

9. Ozawa, T.; Mickle, D. A. G.; Weisel, R. D.; Matsubayashi, K.; Fujii, T.; Fedak, P. W. M.; Koyama, N.; Ikada, Y.; Li, R. K., Tissue-Engineered Grafts Matured in the Right Ventricular Outflow Tract. Cell Transplant. 2004, 13, 169-177. 